\title{
胞内抗体肿瘤基因治疗
}

\section{邵荣光 甄永苏}

(中国医学科学院、中国协和医科大学医药生物技术研究所, 北京 100050. Email: shaor@ public3.bta.net.cn)

自杂交瘤和单克隆抗体技术问世以来，人们一直尝试将该技术用于肿瘤治疗. 抗体工程的 进展使得人们能够对抗体分子进行改造, 研制具有高亲和力和特异结合力的基因工程抗体. 同 时人们对细胞内蛋白传导信号也有了更深入了解，从而可将基因工程抗体导向不同的亚细胞区 室. 上述两项重要进展的结合, 派生出了一项全新的可阻断细胞内重要靶蛋白的胞内抗体 (intracellular antibody)技术. 胞内抗体技术是指应用基因重组技术在非淋巴细胞内表达具有生物 活性的抗体, 并通过对抗体分子进行适当修饰, 使之定向分布于细胞核、细胞浆或某些细胞器 中, 从而特异性干扰或阻断分布于该部位的某些生物大分子的活性或加工、分泌过程, 引起细 胞的一系列生物过程发生改变 ${ }^{[1]}$. 该技术可能对基础医学研究和肿瘤的基因治疗产生深远的影 响.

胞内抗体肿瘤基因治疗是通过基因导入细胞在体内产生抗体，阻断细胞内癌相关蛋白， 间接抑制肿瘤的生长. 现有的研究显示, 胞内抗体的结合靶蛋白及其功能主要有: ( i ) 表皮生 长因子受体 $(\mathrm{EGFR})^{[2]}$ : 在多种肿瘤如脑瘤、肺癌和卵巢癌中过度表达; (ii) 白细胞介素 2 受 体(IL-2R $)^{[3]}$ : 它是一种生长因子受体, 在许多种 $\mathrm{T}$ 细胞和 $\mathrm{B}$ 细胞白血病中均有过度表达; (iii) HER-2(erbB-2) $)^{[4]}$ : 是一种跨膜糖蛋白, 在乳腺癌和卵巢癌等许多肿瘤中均有高表达; (iv) p2 ${ }^{\text {ras }}$ 蛋白 ${ }^{[5]}$ : 突变型的 ras 基因, 广泛存在于许多人类肿瘤, 其蛋白产物 $\mathrm{p} 21^{\mathrm{ras}}$ 是细胞浆中的一种 鸟嘌呤核苷酸结合蛋白, 对细胞的生长、分化起调节作用; (V) c-Myb 蛋白 ${ }^{\left[{ }^{[6]}\right.}$ : 原癌基因 $c-m y b$ 的表达是癌转化的重要因素; ( vi) Bcl-2 蛋白 ${ }^{[7]}$, 是一种抗细胞凋亡蛋白, 能抑制抗肿瘤药物 对细胞的杀伤作用; (vii) 其他: 如人乳头瘤病毒癌相关蛋白以及IV型胶原酶等.

上述胞内抗体的作用靶蛋白与肿瘤的发生、恶性转化及功能等有密切关系, 如 IV 型胶原 酶在肿瘤的侵袭与转移中起重要作用. 王维刚等人 ${ }^{[8]}$ 选用 IV 型胶原酶为靶抗原, 构建抗 $I V$ 型胶 原酶的单链抗体 $(\mathrm{scFv})$, 导入到高转移性人肺巨细胞癌 PG 中, 抗 IV 型胶原酶胞内抗体选择性 与细胞内 $\mathrm{IV}$ 型胶原酶结合, 抑制细胞侵袭转移能力.

对胞内抗体阻断细胞内蛋白的作用机制, 目前研究报道较少. 综合现有的研究结果, 其 作用机制主要包括: ( i ) 抑制或稳定大分子间相互作用, 改变蛋白通常所在区室; (ii) 通过对 配体的修饰使其功能丧失; (iii) 通过底物鳌合或酶活性修饰来改变酶的功能 ${ }^{[9]}$.

胞内抗体对癌相关蛋白的阻断作用主要有以下两种方式:

(1) 胞内抗体在分泌途径上阻断癌相关蛋白. 目前的胞内抗体肿瘤基因治疗研究大部分 都采用这样一个策略, 即针对内质网内的某一特异性肿瘤相关蛋白, 使胞内抗体在内质网内 分泌并与之结合, 阻断其功能活性. 胞内抗体多为单链抗体 ( $\mathrm{scFv}$ ), 为了使 $\mathrm{scFv}$ 滞留于内质网, 通常在单链抗体的氨基端融合一段导肽或在其羧基端引入一段内质网滞留信号. 内质网是多 种生物活性蛋白加工、分泌的通路, 将抗体滞留于内质网管腔或内质网内膜上大大增加了抗 体与靶蛋白相互作用的机会; 内质网本身也是天然抗体组装的场所, 其管腔内存在有利于抗 体形成活性构象所需的辅助因素; 此外, 内质网滞留型抗体的生物活性半衰期要长于细胞浆 
中的胞内抗体 ${ }^{[1]}$. 研究显示, 抗 IL-2R 胞内抗体可有效地阻断 IL-2R 在细胞表面的表达, 其机 制是, 内质网滞留型胞内抗体在内质网中结合 IL-2R, 从而有效阻断其向细胞表面的运输 ${ }^{[3]}$. 最新研究报道, 在真核细胞内质网表达抗 IV 型胶原酶 scFv M97 抗体具有显著抑制 PG 细胞分 泌 $\mathrm{IV}$ 型胶原酶的作用 ${ }^{[8]}$, 结果显示胞内抗体技术可以在蛋白加工、分泌这一关键步骤从根本 上抑制 IV 型胶原酶的活性, 在肿瘤基因治疗中具有重要的应用价值.

（2）胞内抗体在细胞质和细胞核阻断癌相关蛋白. 细胞内表达重组抗体干扰在不同细胞器 表达的肿瘤相关分子, 具有潜在的肿瘤临床治疗前景. 在细胞质中表达胞内抗体一般通过删去免 疫球蛋白的前导序列来完成. 细胞质表达的 $\mathrm{scFv}$ ，包括完整免疫球蛋白被用于在多种细胞中进行 抑制 Ras 信号通道的研究. Cochet 等人 ${ }^{[5]}$ 用胞内表达 $\mathrm{scFv}$ 抑制原癌基因 ras 突变型蛋白功能, 体 外试验证明 $\mathrm{scFv}$ 在肿瘤细胞质表达, 阻断 Ras 介导的激活过程, 引起肿瘤细胞凋亡. 在细胞内表 达抗 p53 的 scFv, 该抗体片段能成功地导向细胞核与 $\mathrm{p} 53$ 结合, 在人肿瘤细胞中部分地恢复突变 型 p53 的转录活性. Caron 等人 ${ }^{[10]}$ 报道, 抗 p53 的 scFv 能够稳定地与 $\mathrm{p} 53$ 结合, 体外试验表明能 恢复某些突变型 $p 53$ 的 DNA 结合活性. 当 $\mathrm{scFv}$ 在 p53 突变的肿瘤细胞中表达时, 能有效地分布 在 p53 缺失的细胞质中, 而当外源性 p53 存在时, $\mathrm{scFv}$ 能有效地分布在细胞核中. 研究表明, 在 cMyb 蛋白表达阳性的细胞 K562 的细胞质和细胞核内表达抗 c-Myb 抗体, 达到了对 c-Myb 癌相关 蛋白的功能性敲除 ${ }^{[6]}$, 为研究 c-Myb 诱导的癌转化分子机制和 c-Myb 依赖性疾病的基因治疗提供了 新思路. 以上研究显示, 直接抗肿瘤基因相关蛋白的胞内单链抗体可能代表一类新的抗肿瘤分子.

研究证明, 胞内抗体具有明显的抗肿瘤活性. 如抗 HER-2 的胞内抗体抑制 HER-2 高表达 卵巢癌细胞的增殖, 并诱导细胞凋亡, 对肿瘤细胞克隆生成具有较强抑制作用 ${ }^{[11]}$. 用编码抗 HER-2 单链抗体的重组腺病毒对移植于裸鼠腹腔的人卵巢癌的治疗结果显示, 胞内表达抗体 可以显著延长动物存活时间. 在 HER-2 高表达肺癌及乳腺癌细胞 ${ }^{[12]}$ 的基因治疗中, 也取得了 类似结果. 用编码抗 HER-2 单链抗体的重组腺病毒对 HER-2 阳性前列腺癌细胞的治疗结果 表明, 人骨髓中前列腺癌得到了有效的清除 ${ }^{[13]}$. Bcl-2 是基因治疗中合适靶点之一, 它通过阻 断多因素引起凋亡的启动而延长细胞的存活, 其表达与肿瘤细胞抗药性及化疗敏感性降低呈 正相关. Piche 等人 ${ }^{[7]}$ 报道, 在人乳腺癌细胞 MCF-7 中胞内表达的抗 Bcl-2 scFv 降低了 Bcl-2 的表达水平, 结果提高了顺铂等药物介导的对该细胞的细胞毒作用. 王维刚等人 ${ }^{[8,9]}$ 的研究表 明, 在真核细胞内质网表达抗IV型胶原酶的 scFv M97 抗体, 细胞内表达 scFv M97 对 PG 细 胞IV型胶原酶分泌、PG 细胞生长及体外侵袭能力具有显著的抑制作用, 并能明显降低 PG 细 胞裸鼠体内成瘤性, 延长动物存活时间. 以上结果提示胞内抗体技术可以在蛋白加工、分泌 这一关键步骤从根本上抑制IV型胶原酶的活性，在肿瘤基因治疗中具有重要的应用价值.

综上所述，胞内抗体可作为一类新分子用于肿瘤的基因治疗，胞内抗体技术可以用作药 理学研究手段分析靶蛋白的功能, 它开辟了一条简单有效的灭活癌相关蛋白的新途径, 可使 人们进一步深入了解癌相关蛋白改变细胞生长和推动癌转化的机制, 该技术将在基础医学研 究、癌转化机制研究和肿瘤等疾病的基因治疗中发挥重要作用. 以胞内抗体为基础的肿瘤靶 向基因治疗的研究进展将使肿瘤基因治疗的理论和临床研究水平上升到一个新的高度.

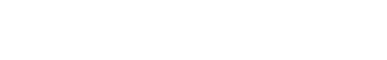

1 王维刚, 甄永苏. 以抗体为基础的肿瘤靶向治疗和基因治疗. 药学学报, 1999, 34(10): 795 800 
2 Goldman C K, Rogers B E, Douglas J T, et al. Targeted gene delivery to Kaposi's sarcoma cells via the fibroblast growth factor receptor. Cancer Res, 1997, 57(8): $1447 \sim 1451$

3 Richardson J H, Sodroski J G, Waldmann T A, et al. Phenotypic knockout of the high-affinity human interleukin 2 receptor by intracellular single-chain antibodies against the alpha subunit of the receptor. Proc Natl Acad Sci USA, 1995, 92(8): $3137 \sim 3141$

4 Foster B J, Kern J A. HER2-targeted gene transfer. Human Gene Ther, 1997, 8(6): $719 \sim 727$

5 Cochet $\mathrm{O}$, Gruel N, Fridman W H, et al. Ras and p53 intracellular targeting with recombinant single-chain Fv (scFv) fragments: a novel approach for cancer therapy? Cancer Detect Prev, 1999, 23(6): $506 \sim 510$

6 Kasono K, Piche A, Xiang J, et al. Functional knock-out of $c$-myb by an intracellular anti-c-Myb single-chain antibody. Biochem Biophy Res Commun, 1998, 251(1): $124 \sim 130$

7 Piche A, Grim J, Rancourt C, et al. Modulation of Bcl-2 protein levels by an intracellular anti-Bcl-2 single-chain antibody increases drug-induced cytotoxicity in the breast cancer cell line MCF-7. Cancer Res, 1998, 58(10): $2134 \sim 2140$

8 王维刚, 张胜华, 李 毅, 等. 以 IV 型胶原酶为靶点的细胞内表达单链抗体的抗肿瘤作用. 中国科学, C辑, 2000, 30(2): $123 \sim 130$

9 Richardson J H, Marasco W A. Intracellular antibodies: development and therapeutic potential. Trends Biotechnol, 1995, 13(8): $306 \sim 310$

10 Caron de Fromentel C, Gruel N, Venot C, et al. Restoration of transcriptional activity of p53 mutants in human tumour cells by intracellular expression of anti-p53 single chain Fv fragments. Oncogene, 1999, 18(2): $551 \sim 557$

11 Deshane J, Siegal G P, Alvarez R D, et al. Targeted tumor killing via an intracellular antibody against erbB-2. J Clin Invest, 1995, 96(6): $2980 \sim 2989$

12 Wright M, Grim J, Deshane J, et al. An intracellular anti-erbB-2 single-chain antibody is specifically cytotoxic to human breast carcinoma cells overexpressing erbB-2. Gene Ther, 1997, 4(4): $317 \sim 322$

13 Kim M, Wright M, Deshane J, et al. A novel gene therapy strategy for elimination of prostate carcinoma cells from human bone marrow. Human Gene Ther, 1997, 8(2): $157 \sim 170$

(2000-03-10 收稿, 2000-04-20 收修改稿) 